\title{
Modelling Superconducting Nanowire Single Photon Detectors in a Waveguide-Based Ring Resonator
}

\author{
Gerardo E. Villarreal-Garcia, Nicola A. Tyler, Jorge Barreto, Damien Bonneau, Döndü Sahin, Jeremy L. O’Brien \\ and Mark G. Thompson \\ Centre for Quantum Photonics, H. H. Wills Physics Laboratory, University of Bristol, Bristol, BS8 1TL, United Kingdom \\ g.villarreal@bristol.ac.uk
}

\begin{abstract}
We present an analysis of a single photon detector system capable of achieving near-unity detection efficiency. It consist of waveguidecoupled superconducting nanowires as short as $1 \mu \mathrm{m}$ embedded in a racetrack resonator.
\end{abstract}

\section{INTRODUCTION}

Single photon detectors (SPD) are key components for quantum photonic applications such as quantum information processing [1]. Superconducting nanowire SPDs (SNSPDs) are one of the best allaround candidates for single photon detection, offering high efficiency [2] along with low dark-count rates [3] and low jitter [4]. SNSPDs are compatible with planar fabrication technologies, and can be made using thin films of niobium-based materials, such as niobium nitride $(\mathrm{NbN})$, which has a superconducting transition well above $4.2 \mathrm{~K}$, allowing the use of both ${ }^{4} \mathrm{He}$-based and cryogen-free cryostats.

Despite their many advantages, high-efficiency NbN SNSPDs are subject to very low fabrication yields due to defects in the thin $\mathrm{NbN}$ films [5][6], which forces researchers to hand-pick the best devices from a batch [7]. The longer the SNSPD, the higher the probability of having defects. Although it is true that defects can be minimized by using amorphous superconductors which are less prone to structural inhomogeneities such as WSi [8], MoSi [9] and MoGe [10], it comes at the cost of having even lower operating temperatures and lower signal-to-noise ratios.

An alternative approach to the vertical coupling of traditional SNSPDs is the integration of the superconducting nanowires in waveguide structures [11], where the photon travels along a waveguide with an SNSPD directly above, leading to an increased interaction length of photon and nanowire, and therefore to higher

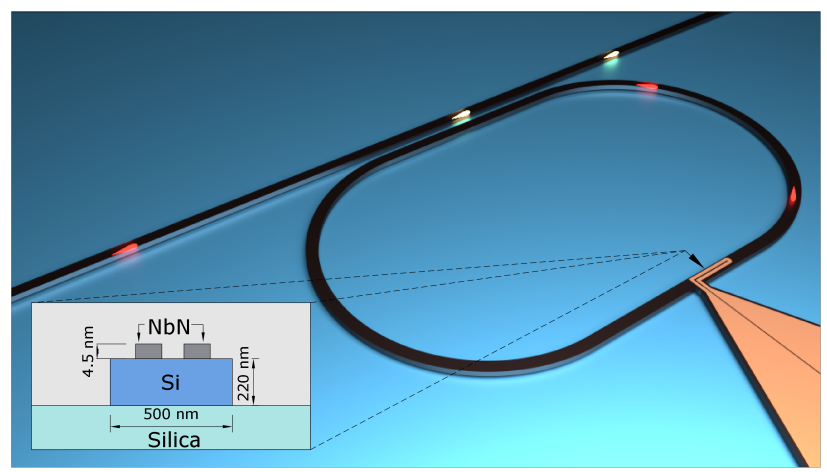

Fig. 1. SNSPD inside SOI waveguide racetrack resonator cavity.

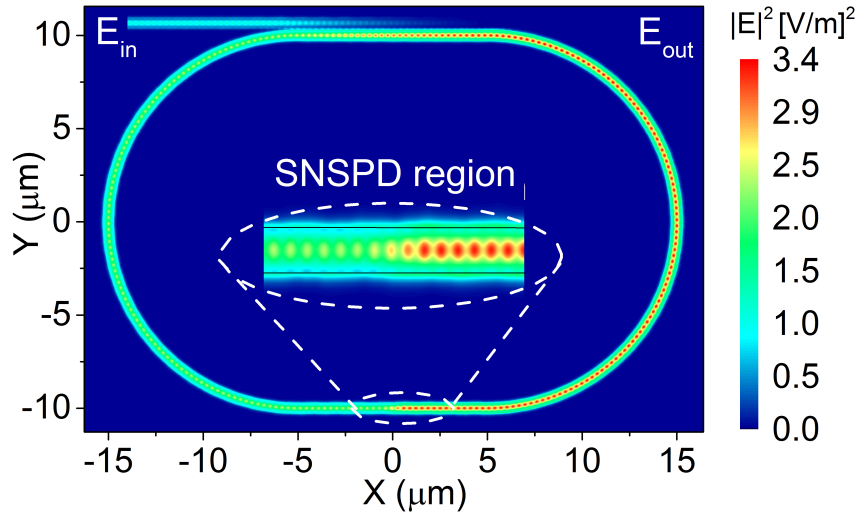

Fig. 2. Intensity plot for quasi-fundamental TE mode for a critically coupled cavity system, simulated using varFDTD [14]. The SNSPD used in this simulation is $1.13 \mu \mathrm{m}$ long, the cavity length is $82.83 \mu \mathrm{m}$ and the wavelength $1553 \mathrm{~nm}$

efficiencies with shorter nanowires [12]. Furthermore, placing waveguide coupled SNSPDs in a cavity has been shown to enhance the interaction time between detector and photon, leading to an increase in detection efficiency. Recently, a 96\% efficient SNSPD has been reported using a nanowire as short as $8.5 \mu \mathrm{m}$ implemented in a photonic crystal cavity [13]. In this work we theoretically study a waveguide coupled SNSPD in a ring resonator cavity, in order to further reduce the detector length while reaching near unity detection efficiencies.

\section{DESign AND SIMUlation RESUlts}

The design we propose is presented in Fig. 1, which consists on an integrated SNSPD patterned as two $4.5 \mathrm{~nm}$ thick, $100 \mathrm{~nm}$ wide and $100 \mathrm{~nm}$ pitch $\mathrm{NbN}$ nanowires deposited on a racetrack ring resonator which is coupled to a a silicon-on-insulator (SOI) waveguide, with dimensions of $0.5 \mu \mathrm{m}$ by $0.22 \mu \mathrm{m}$. The transmission from the waveguide into the cavity depends not only on geometrical factors such as the coupling coefficient between the ring and the waveguide but also on the losses inside the cavity and the wavelength of the optical input. When the losses in the cavity equal the coupling coefficient, critical coupling is achieved leading to $100 \%$ of the input light being coupled into the ring, which can then be absorbed by the SNSPD or scattered by the racetrack waveguide. Because of the interaction time between the detector and the optical input increases, the SNSPD detection efficiency is improved.

In Fig. 2 a varFDTD simulation is presented with an example geometry which was designed to work close to critical coupling for $1553 \mathrm{~nm}$. The length of the racetrack is $82.83 \mu \mathrm{m}$, while the SNSPD has a length of $1.13 \mu \mathrm{m}$ and a coupling coefficient for the ring of 

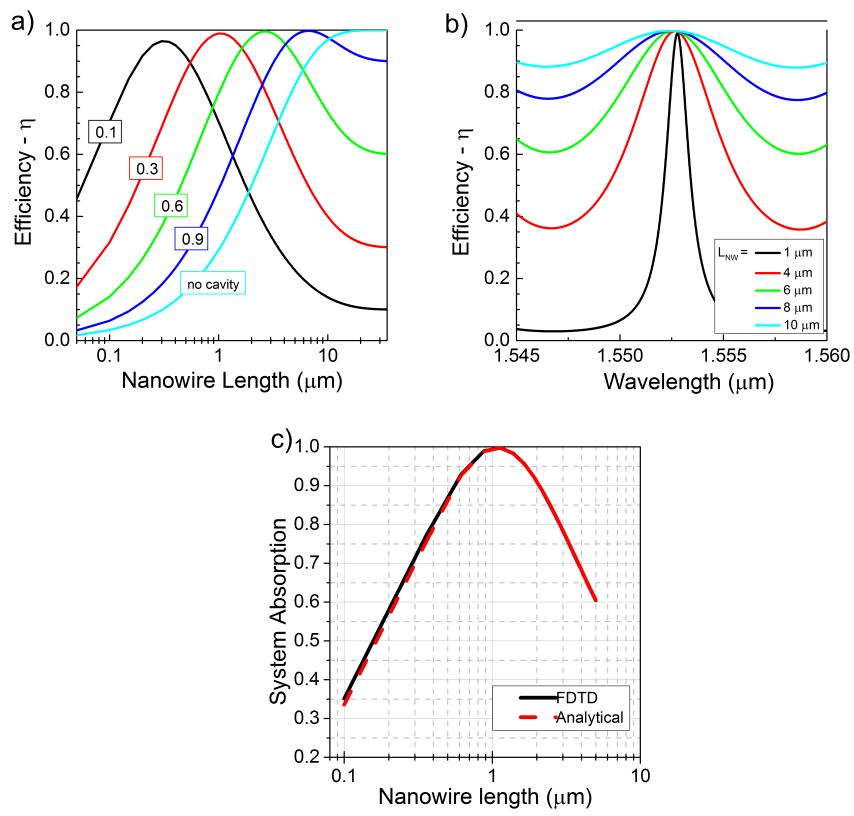

Fig. 3. a)Detection efficiency as function of the nanowire length. Efficiencies close to unity can be achieved if the coupling coefficient (shown on different colors) is tuned properly. b) Wavelength response of the detection efficiency for a CW input at critical coupling for different nanowire lengths. c)Comparison between FDTD simulation and analytical calculations of the total system absorption versus nanowire length.

0.31 . The simulation was done using a commercial-grade simulator eigenmode solver and varFDTD propagator [14].

Numerical simulations for the absorption in the nanowire region were contrasted with an analytical model assuming $100 \%$ of internal quantum efficiency. The dependency between detection efficiency and nanowire length for different coupling coefficients is shown in Fig. 3(a) including the particular case where the detector is deposited on a straight waveguide without cavity. Efficiencies close to unity can be achieved with shorter nanowire lengths than in the no-cavity instance due to increased interaction time between the photon and the nanowire. The simulated cavity length is $83 \mu \mathrm{m}$, the system is phasematched around $1553 \mathrm{~nm}$ and $-2 \mathrm{~dB} / \mathrm{cm}$ waveguide loss is assumed. The performance of the detector follows the cavity response but it is affected by the length of the nanowire region, with sharper peaks for shorter nanowires as can be seen in Fig. 3(b). Additionally, we have modelled the system for a pulsed input with Gaussian frequency distribution. For pulses with $0.1 \mathrm{~nm}$ of full width at half maximum efficiencies as high as $98.7 \%$ with a nanowire region as short as $1 \mu \mathrm{m}$ inside a $83 \mu \mathrm{m}$ racetrack, and $99.9 \%$ efficiency for a $10 \mu \mathrm{m}$ nanowire region and a $30 \mu \mathrm{m}$ racetrack have been found.

Finally, the results for a racetrack resonator of $83 \mu \mathrm{m}$ length and a coupling coefficient of 0.31 obtained with our analytical model for $\mathrm{CW}$ input on resonance at $1553 \mathrm{~nm}$, are compared to a numerical simulation in Fig. 3(c) and are in strong agreement. Critical coupling is achieved for a nanowire length of $1.13 \mu \mathrm{m}$ with a total system absorption $^{1}$ maximum of $99.76 \%$ which can be seen in Fig. 2. Around this point, the detection probability is maximized.

\footnotetext{
${ }^{1}$ Total system absorption includes absorption by the detector and light scattered by silicon in the cavity
}

\section{CONCLUSION}

In conclusion, we presented a numerical simulation of a waveguide coupled SNSPD deposited on a SOI racetrack ring which can be designed to obtain detection efficiencies close to unity for nanowire lengths as short as $1 \mu \mathrm{m}$ using standard CMOS-compatible photonic elements. The option of using short nanowires will not only be able address the problem of low fabrication yield, but it will also bring new possibilities in terms of scalability. Additionally, the wavelengthdependent behaviour of this type of detectors opens the door for applications such as custom-designed spectrometers.

\section{REFERENCES}

[1] E. Knill, R. Laflamme, and G. J. Milburn, "A scheme for efficient quantum computation with linear optics," Nature, vol. 409, no. 6816 , pp. 46-52, 2001.

[2] M. Eisaman, J. Fan, A. Migdall, and S. Polyakov, "Invited review article: Single-photon sources and detectors," Review of Scientific Instruments, vol. 82, no. 7, p. $071101,2011$.

[3] C. Schuck, W. H. Pernice, and H. X. Tang, "Waveguide integrated low noise nbtin nanowire single-photon detectors with milli-hz dark count rate," Scientific reports, vol. 3, 2013.

[4] R. H. Hadfield, "Single-photon detectors for optical quantum information applications," Nature photonics, vol. 3, no. 12, pp. 696-705, 2009.

[5] C. Constancias, R. E. De Lamaëstre, O. L. P. Cavalier, J. Villegier et al., "Patterning issues in superconducting nanowire single photon detector fabrication," Journal of Vacuum Science \& Technology B, vol. 25, no. 6, pp. 2041-2044, 2007.

[6] G. Gol'tsman, O. Okunev, G. Chulkova, A. Lipatov, A. Dzardanov, K. Smirnov, A. Semenov, B. Voronov, C. Williams, and R. Sobolewski, "Fabrication and properties of an ultrafast nbn hot-electron single-photon detector," Applied Superconductivity, IEEE Transactions on, vol. 11, no. 1, pp. 574-577, 2001.

[7] M. Hofherr, D. Rall, K. Ilin, M. Siegel, A. Semenov, H.-W. Hübers, and N. Gippius, "Intrinsic detection efficiency of superconducting nanowire single-photon detectors with different thicknesses," Journal of Applied Physics, vol. 108, no. 1, p. 014507, 2010.

[8] F. Marsili, V. Verma, J. Stern, S. Harrington, A. Lita, T. Gerrits, I. Vayshenker, B. Baek, M. Shaw, R. Mirin et al., "Detecting single infrared photons with 93\% system efficiency," Nature Photonics, vol. 7, no. 3, pp. 210-214, 2013.

[9] Y. P. Korneeva, M. Y. Mikhailov, Y. P. Pershin, N. Manova, A. Divochiy, Y. B. Vakhtomin, A. Korneev, K. Smirnov, A. Sivakov, A. Y. Devizenko et al., "Superconducting single-photon detector made of mosi film," Superconductor Science and Technology, vol. 27, no. 9, p. 095012, 2014.

[10] V. Verma, A. Lita, M. Vissers, F. Marsili, D. Pappas, R. Mirin, and S. W. Nam, "Superconducting nanowire single photon detectors fabricated from an amorphous mo0. 75ge0. 25 thin film," Applied Physics Letters, vol. 105, no. 2, p. 022602, 2014.

[11] E. A. Dauler, M. E. Grein, A. J. Kerman, F. Marsili, S. Miki, S. W. Nam, M. D. Shaw, H. Terai, V. B. Verma, and T. Yamashita, "Review of superconducting nanowire single-photon detector system design options and demonstrated performance," Optical Engineering, vol. 53, no. 8, pp. 081 907-081 907, 2014.

[12] W. H. Pernice, C. Schuck, O. Minaeva, M. Li, G. Goltsman, A. Sergienko, and H. Tang, "High-speed and high-efficiency travelling wave single-photon detectors embedded in nanophotonic circuits," $\mathrm{Na}$ ture communications, vol. 3, p. 1325, 2012.

[13] M. K. Akhlaghi, E. Schelew, and J. F. Young, "Waveguide integrated superconducting single-photon detectors implemented as near-perfect absorbers of coherent radiation," Nature Communications, vol. 6, 2015.

[14] Lumerical Solutions, Inc. http://www.lumerical.com/tcadproducts/mode/. 